\title{
Pattern formation in a rotating suspension of non-Brownian settling particles
}

\author{
W. R. Matson, ${ }^{1}$ B. J. Ackerson, ${ }^{1}$ and P. Tong ${ }^{1,2}$ \\ ${ }^{1}$ Department of Physics, Oklahoma State University, Stillwater, Oklahoma 74078 \\ ${ }^{2}$ Department of Physics, Hong Kong University of Science and Technology, Clear Water Bay, Kowloon, Hong Kong, China
}

(Received 17 April 2002; published 14 May 2003)

\begin{abstract}
We report band formation and other pattern formation for a settling suspension of uniform non-Brownian particles in a completely filled horizontal rotating cylinder. The system shows a series of sharp pattern changes that are mapped out as a function of the rotation period and suspension viscosity. The experiment suggests that a large number of patterns and rich dynamics result from the interplay among the viscous drag, and gravitational and centrifugal forces.
\end{abstract}

DOI: 10.1103/PhysRevE.67.050301

PACS number(s): 45.70.Qj, 05.65.+b, 83.10.Pp, 83.80.Hj

Nonequilibrium systems often organize into interesting spatiotemporal structures or patterns. Examples include the patterns in pure fluid flow systems, such as the RayleighBénard convection in a vertical temperature gradient, the Taylor-Couette flow between two concentric rotating cylinders, and parametric wave instabilities on a liquid-air interface that is rigidly oscillated in the vertical direction [1]. The fluid in a partially filled, horizontal, rotating cylinder (a coating flow) also shows pattern formation [2]. In recent years, pattern formation in externally excited granular flow systems received much attention. Small differences either in grain size or density lead to grain segregation and pattern formation when the particles flow or are shaken. Examples include radial segregation and axial banding of binary granular mixtures when they are partially filled in a horizontal rotating cylinder [3]. While the granular flows can generate many patterns, surprisingly similar to those found in ordinary fluids, our current understanding of granular flows is far less than that for simple fluid flows.

The hydrodynamics of uniform non-Brownian hard spheres suspended in a Newtonian fluid fills the gap between the two limiting cases. Although the single-particle motion is known with high precision, the collective behavior of the particles often shows interesting but unexpected features [4]. Because of the long ranged hydrodynamic interactions, the particle configuration and structure formation are strongly coupled to the flow field, making the suspension dynamics an interesting and challenging problem in statistical physics. Recent experiments [5] in a horizontal rotating cylinder or Couette device partially filled with a suspension of either neutrally buoyant or heavy particles revealed that the particles again separate into a series of axial bands stacked along the horizontal axis. While generally accepted explanations are yet to obtain, surfaces are thought to be important to structure formation in particle laden systems, either the sliding granular surface or the flowing fluid-air interface.

In this paper, we report band formation and other pattern formations for a settling suspension of uniform nonBrownian particles in a completely filled horizontal rotating cylinder. Here there is no surface responsible for pattern formation, yet the system shows a series of sharp pattern changes. Because of the inbalance between the solvent pressure gradient and the centrifugal force due to rotation, an individual (heavy) particle should not stay suspended in a rotating cylinder indefinitely. The particle will spiral outwards continuously to the cell wall [6]. Nevertheless, the collective behavior of the rotating suspension is found to be qualitatively different from the single-particle dynamics. Figure 1 shows the "phase diagram" of this system as a function of the rotation period $T_{R}=2 \pi / \omega$ and solution viscosity $\eta$. There are nine different steady states observed, and they are distinguished in the experiment by their unique flow patterns and particle distributions.

The experiment utilizes a horizontal Plexiglas cylinder with an internal diameter $2 R=1.85 \mathrm{~cm}$ and adjustable length $L$ varied from 22.8 to $1.2 \mathrm{~cm}$. The cylinder is completely filled with an aqueous solution of glycerin with mass concentration of glycerin ranged between 40 and $80 \mathrm{wt} \%$. The corresponding solution viscosity $\eta$ varies from 5 to $75 \mathrm{cp}$ at

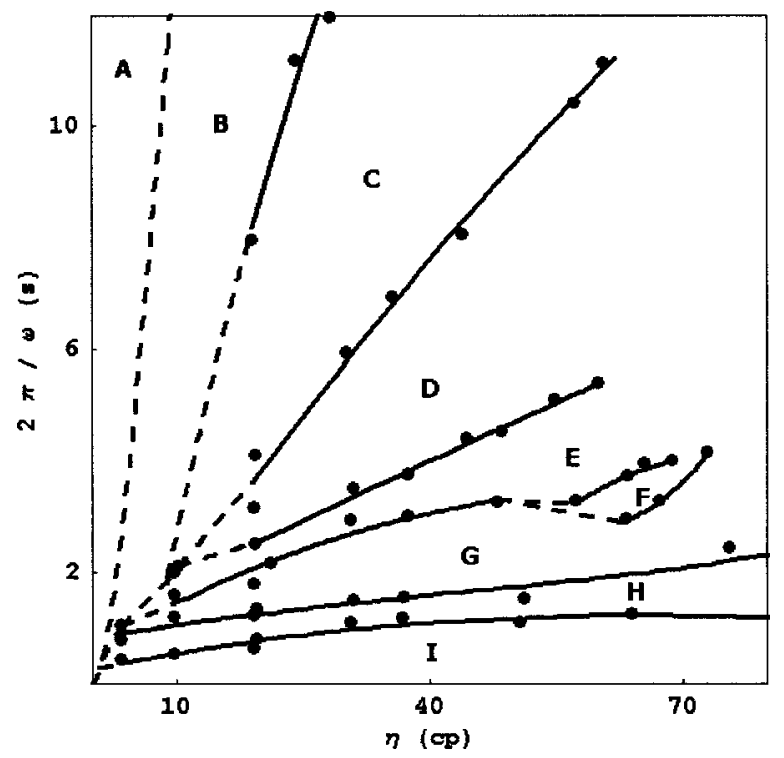

FIG. 1. Part of the phase diagram as a function of $2 \pi / \omega$ and $\eta$. The symbols are experimentally determined data points and the solid lines are spline-fitted smooth curves for the phase boundaries. The dashed lines are the extrapolated curves from the fit. Region $A$, dense particle bed cascade; region $B$, fingering; region $C$, fingerband coexistence; region $D$, bands; region $E$, band loss or bandrigid body coexistence; region $F$, rigid body rotation; region $G$, asymmetric segregation; region $H$, asymmetric bands; region $I$, centrifugally dominated collapse. 
room temperature $\left(\sim 20^{\circ} \mathrm{C}\right)$. Uniform glass spheres of $a$ $=100 \mu \mathrm{m}$ in radius and $\rho_{p}=2.4 \mathrm{~g} / \mathrm{cm}^{3}$ in density are dispersed in the solution with a fixed volume fraction $\phi$ $=0.0229$. The size variation of the particles is less than $8 \%$. A small amount of detergent is added into the solution to prevent particle aggregation. The cylinder rotates around its axis of symmetry ( $z$ axis) and is driven by a thermally isolated stepper motor with $2.5 \times 10^{4}$ steps/rotation. An indexer is constructed to provide a stable pulse train (1.0 $\times 10^{-6} \mathrm{~s} /$ pulse) to the stepper driver. The rotating cylinder and the stepper motor are mounted on an aluminum rack, which can be leveled with high accuracy.

In the experiment, we use a standard video imaging technique to record the motion and spatial distribution of the particles. A video charge-coupled device camera records particle images for different cross-sectional planes of the cylinder ( $r-\theta$ planes) and in the $\theta-z$ planes. To facilitate the imaging in the $r-\theta$ plane (end view), we make a separate short cylinder $(1.3 \mathrm{~cm}$ in length) with the same diameter, but having a transparent end window for video imaging. A sheet of white light illuminates the $r-\theta$ plane. For the imaging in the $\theta-z$ planes (side view), backlit illumination is used. The particle trajectories are visible as streaks in superposed consecutive images. To vary the contrast of the particle images, we use both blue and translucent white glass spheres.

Because of the density difference $\Delta \rho=\rho_{p}-\rho_{s}$ between the particle and the solvent, the particles settle in the rotating solution. The Stokes velocity in the $80 \mathrm{wt} \%$ glycerin solution $\quad\left(\eta \simeq 58 \mathrm{cp}, \rho_{s} \simeq 1.2 \mathrm{~g} / \mathrm{cm}^{3}\right) \quad$ is $\quad U_{0}=\frac{2}{9} a^{2} \Delta \rho g /$ $\eta \simeq 0.45 \mathrm{~mm} / \mathrm{s}$, giving a total settling time across the tube radius $\tau_{s} \simeq 20.5 \mathrm{~s}$. The Peclet number given by $\mathrm{Pe}$ $=U_{0} a / D_{0} \simeq 1.1 \times 10^{9}$, where $D_{0}$ is the Stokes-Einstein diffusion constant, implies negligible Brownian motion. The Reynolds numbers based on particle radius, $\mathrm{Re}$ $=2 a U_{0} \rho_{s} / \eta \simeq 2 \times 10^{-3}$, and on tube radius, $\operatorname{Re}$ $=R U_{0} \rho_{s} / \eta \simeq 0.09$, imply negligible inertial effects. The Reynolds number of the rotational flow itself is $\mathrm{Re}$ $=\omega R^{2} / \eta \simeq 32$ for the maximum rotation rate $\omega \simeq 17.4 \mathrm{~s}^{-1}$. Nevertheless, the rotating suspension of the non-Brownian particles exhibits sharp transitions to various spatial structures in both the concentration and velocity fields.

We now discuss the pattern formation observed with increasing $\omega$ when $\eta$ is in the range $5-75 \mathrm{cp}$. When $\omega$ is zero, the particles lie on the bottom as a close random packed sediment. As $\omega$ increases (Fig. 1, region $A$ ), the particles form the dense fluidized bed that resembles cascade flow in granular systems [3] and coating flow in pure fluid systems [2]. The bed evidences clockwise circulation going up along the rising cell surface and down at the bed solvent interface [Fig. 2(a)]. This gravity driven flow produces a counterclockwise rotation of the pure solvent at the cell center.

At higher $\omega$ (Fig. 1, region $B$ ), the top leading edge of the particle bed moves into the upper half of the cell. Particles are injected into the pure solvent region and fall forming a curtainlike structure that bends back to the bed due to the counterclockwise flow field. At the lower trailing edge of the bed, particles are also injected where the counterclockwise flow meets the wall driven clockwise flow [Fig. 2(b)]. Par-

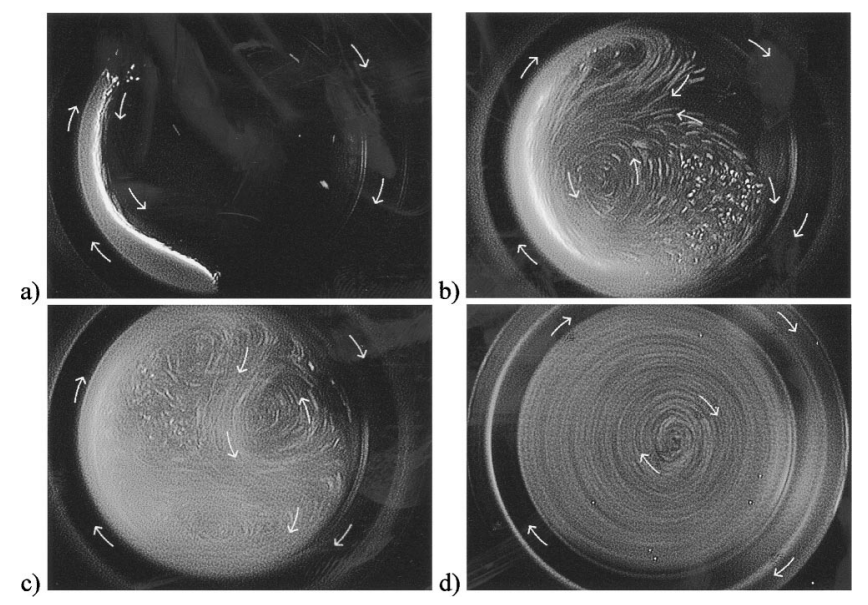

FIG. 2. End view of a short rotating cylinder. For clarity, the contrast of the pictures is reversed with white streaks against a dark background showing the particle trajectories. (a) Dense particle bed cascade with some ejection from the leading and trailing edges of the bed, (b) counterclockwise rotation region now populated by particles, (c) unstable flow with significant three-dimensional components, and (d) clockwise-only trajectories in the band region.

ticles within the curtain form groups of chainlike structures. At first, only two or three particles move downward together. The number of particles per chain, the chain diameter, chain length, and chain number increase with $\omega$. We call this "finger formation." When the particles on the leading edge of the dense particle bed reach the top ceiling of the cell, the finger width is 10-20 particles across with a length to width ratio of $\sim 25$. The settling speed is approximately five times larger than the Stokes velocity $U_{0}$. These large-scale structures randomly perturb the formerly two-dimensional flow structure.

When $\omega$ is increased to region $C$, the three-dimensional flows begin to interact to form an unsteady collection of bands evidencing significant particle density variation and secondary flow structure along the axis of rotation. As $\omega$ approaches the dynamic phase boundary between regions $C$ and $D$, the band structure becomes increasingly stable compared to the coexisting fingering. At the same time, the counterclockwise circulation becomes excluded in favor of more clockwise particle trajectories [Fig. 2(c)].

When $\omega$ is further increased to region $D$, the secondary flow patterns stabilize to form bands [Fig. 3(a)]. The position of the bands remains fixed and stable for a given $\omega$ with fairly uniform spacing, except near the cylinder ends. The typical spacing between the bands is $1.2 \pm 0.1$ times the tube diameter and is independent of other control parameters, such as the solution viscosity, the tube length, and the particle size. In region $D$ the particle trajectories become exclusively clockwise [Fig. 2(d)]. Note that the "center" of the particle trajectories is not coincident with the axis of rotation. The trajectory center follows a zigzag path, being closer to the upgoing wall for the $r-\theta$ planes with the highest particle densities and closest to the downgoing wall for the smallest particle densities. Particles are drawn into the dense band regions by secondary flows. Evidently, this increased particle concentration produces a larger downward velocity and reinforces the secondary flow. 


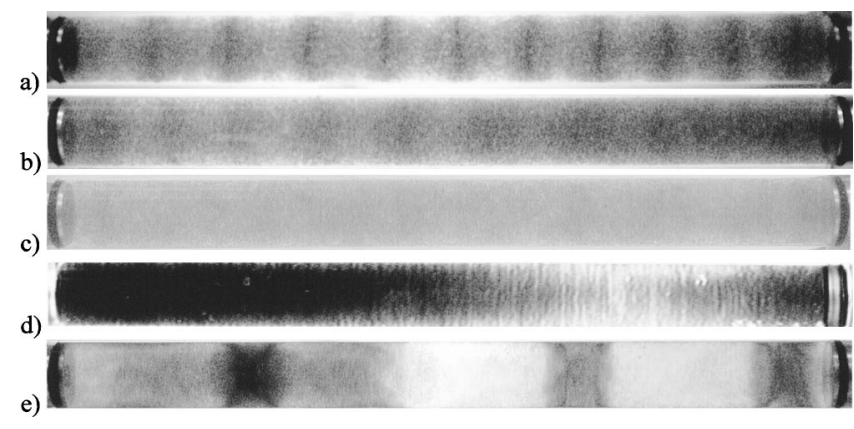

FIG. 3. Side view of a long rotating cylinder. Darker regions have more particles. (a) Bands observed in phase $D$, (b) band decay in phase $E$ with missing bands on the right side of the tube, (c) uniform particle distribution in phase $F$, (d) asymmetric particle segregation in phase $G$, and (e) asymmetric bands observed in phase $H$.

At even higher $\omega$ (Fig. 1, region $E$ ), the bands retreat radially from the cell walls towards the axis of rotation. This occurs mostly in the high viscosity region $(\eta>38 \mathrm{cp})$. The retreat of the concentrated band regions is uniform along the rotation axis and the spatial distribution of the bands remains unchanged. In the outer "shell" region, the particles rigidly rotate with the cylinder and the spatial distribution is uniform. As $\omega$ approaches the high- $\omega$ boundary of region $E$, the bands drop off at the tube ends [Fig. 3(b)]. For high viscosity solutions (near region $F$ ), the drop-off process continues with increasing $\omega$ until all the bands disappear from the entire tube. Evidently, the secondary circulation cannot keep up with the increased cell rotation. The whole suspension then remains uniform [Fig. 3(c)] and undergoes a "rigid body rotation." This small region is labeled $F$ in Fig. 1. For low viscosity solutions, the drop-off process is terminated when the system changes to a new flow state.

Further increase in $\omega$ (Fig. 1, region $G$ ) results in a largescale nonuniform particle distribution. As shown in Fig. 3(d), the particles migrate to both ends of the tube and form two cone-shaped (dark) regions facing towards each other and extending along the $z$ axis. When viewed from the side, the two cone-shaped regions are surrounded by an unpopulated (light) region, whose location is asymmetric being on one side of the tube. Tipping the rotation axis from the horizontal results in particle migration to the lower end when $\omega$ is small. We leveled the tube to avoid this gravitational effect.

As $\omega$ increases further (Fig. 1, region $H$ ), the wide coneshaped region contracts to form from one to three concentrated bands having a width of $2-3 \mathrm{~cm}$ [Fig. 3(e)]. The location of the band regions appears random and they evidence no interband interaction. Each band is composed of a very dense, concave-lens-shaped core region surrounded by a less dense cloud of particles. Particles inside each band region are ejected periodically in groups parallel to, but slightly displaced from, the axis of rotation. The ejected particles travel along the axis of rotation in two distinct ways. (1) They spiral about the axis of rotation for some distance and then retreat relatively quickly along this axis. (2) They spiral outwards about the axis of rotation in a helical path of increas- ing radius. When the particles nearly reach the cell wall, their velocity is altered rapidly and the particles are injected back into the originating band near the bottom of the cylinder. In this way, the particles appear to travel on closed trajectories keeping the total mass of the band constant.

Finally, increasing $\omega$ (Fig. 1, region $I$ ) causes the particles ejected from the bands in region $H$ to spiral outwards to the cell wall without returning to the band region. In a relatively short period of time, this centrifugally dominated flow coats the cell wall with particles and eliminates all the previous band structures.

While the above discussion on the phase behavior of the system is largely descriptive, the actual determination of each phase is based on the sudden changes of both the concentration and flow fields. By watching the motion of the particles over a period of time, one can distinguish these dynamic phases accurately. The observed effects are drastic, robust, and have been reproduced many times in various cells and under different sample conditions. To establish the phase boundaries, we change $\omega$ in both directions and examine the flow patterns using different viewing techniques (end and side views). The highly accurate rotation control produced sharp phase boundaries with no hysteresis. In certain phase changes, such as from phase $H$ to phase $I$, the transition is so sharp that it lies beyond our resolution limit for $\omega$.

Figure 1 shows that certain phases disappear as $\eta$ decreases. The first phase to disappear is the rigid body rotation (phase $F$ ), which ceases to be observed below $\sim 57 \mathrm{cp}$. The band state (phase $D$ ) disappears when $\eta<10 \mathrm{cp}$. While most phase boundaries are nonlinear functions of $\eta$, the band phase boundaries (region $D$ ) are well described by linear functions of $\eta$. In particular, the onset rotation period $T_{c}(s)=T_{0}+0.157 \eta(\mathrm{cp})$ represents the upper boundary of phase $D$. Here, $T_{0}=1.01 \mathrm{~s}$ is the offset rotation period at $\eta=0$.

An important question one might ask is what are the dimensionless control parameters for the system? We have considered various combinations of relevant velocity, time, and length scales based on the single-particle dynamics, but were unable to verify them experimentally. New experiments are underway looking at the influence of other experimental parameters, such as particle size, concentration and tube diameter, on the phase diagram shown in Fig. 1. The present experiment suggests that the large number of patterns and rich dynamics found in the system come from the interplay among the viscous drag, and gravitational and centrifugal forces. It is likely that the dynamics of the system is controlled by more than one dimensionless parameters.

The following are some estimates for the characteristic times involved in the rotating system. Using the settling time across the tube radius $\tau_{s}=R / U_{0}$ as a scaling factor, we find that the onset of the band formation is described by the condition $\left(T_{c}-T_{0}\right) / \tau_{s} \simeq 0.5$. The settling is balanced by the lift of the upward moving wall. Note that this time ratio is proportional to that of the Stokes velocity $U_{0}$ to the tube rotational velocity $\omega R$. By equating the acceleration of gravity $g$ with the centripetal acceleration $\omega_{0}^{2} R$ at the cell wall, we get another characteristic time $2 \pi / \omega_{0} \simeq 0.2$, which is indepen- 
dent of $\eta$ and approximates the phase boundary between regions $H$ and $I$ at the low- $\eta$ limit.

Finally, we calculate the traveling time for a single particle to settle radially from $R / 2$ to $R$ in a rotating solvent. By equating the centrifugal force with the viscous drag on the particle, we have $T_{s}$ (days $)=(g \ln 2) /\left(U_{0} \omega^{2}\right) \simeq 0.175 / \omega^{2}$ with $\omega$ measured in rad/s. For $\omega \simeq 0.416 \mathrm{rad} / \mathrm{s}$, the settling time is one day. For larger $\omega$, the time is correspondingly smaller. The various steady phases have been run stably for weeks. Thus, it is difficult to understand which force suspends the particles for the rigid body phase (region $F$ ) in the presence of centrifugal forces. We note, however, that the above simple considerations are based on the single-particle dynamics, but the patterns observed in the experiment result from the collective motion of the particles, which is qualitatively different from the single-particle dynamics. A further theoretical analysis is needed.

We thank Anthony J. C. Ladd for useful discussions. The assistance of Mike Lucas and his team in fabricating the experimental apparatus is gratefully acknowledged. This work was supported in part by NASA under Grant No. NAG3-1852 and by NSF under Grant No. DMR-0071323.
[1] M.C. Cross and P.C. Hohenberg, Rev. Mod. Phys. 65, 851 (1993).

[2] S.T. Thoroddsen and L. Mahadevan, Exp. Fluids 23, 1 (1997); A.E. Hosoi and L. Mahadevan, Phys. Fluids 11, 97 (1999).

[3] Y. Oyama, Bull. Inst. Phys. Chem. Res. (Tokyo) Rep. 5, 600 (1939); O. Zik et al., Phys. Rev. Lett. 73, 644 (1994); K.M. Hill and J. Kakalios, Phys. Rev. E 49, R3610 (1994); 52, 4393 (1995).
[4] M.C. Roco, Particulate Two-Phase Flow (ButterworthHeinemann, Boston, MA, 1996); C. Voltz et al., Phys. Rev. E 65, 011404 (2001).

[5] M. Tirumkudulu et al., Phys. Fluids 11, 507 (1999); M. Tirumkudulu et al., ibid. 12, 1615 (2000); P.J. Thomas et al., ibid. 13, 2720 (2001).

[6] G.O. Roberts et al., J. Fluid Mech. 229, 555 (1991). 